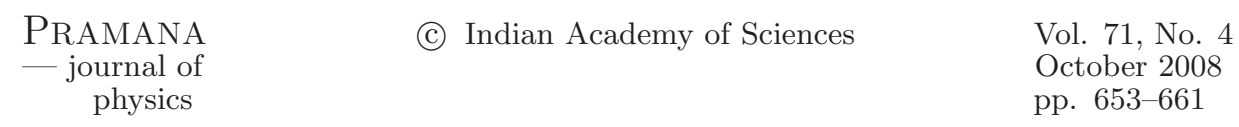

\title{
Recent improvements in the methodology of neutron imaging
}

\author{
EBERHARD H LEHMANN \\ Spallation Neutron Source Division, Neutron Imaging \& Activation Group, Paul Scherrer \\ Institut, CH-5232 Villigen PSI, Switzerland \\ E-mail: eberhard.lehmann@psi.ch
}

\begin{abstract}
The focus of this article is on further improvements of methods in neutron imaging: the increased spatial resolution for microtomography and options for energyselective neutron imaging. Before going into details, some common statements are given in respect to the state-of-the-art in neutron imaging. A relation to the X-ray methods is mentioned, where complementary results are obtained. The potential for the energy selection is of particular interest for future installations at the new pulsed sources, based on spallation (SNS, J-PARC, ISIS-TS2). First results from preliminary studies look very promising for future material and industrial research. Therefore, statements about the set-up of the best possible imaging systems are included in the article.
\end{abstract}

Keywords. Neutron imaging; energy-selection; time-of-flight; neutron tomography; phase-contrast imaging; Bragg edges.

PACS Nos 87.59.F; 61.12.Yp; 07.05.Pj

\section{Introduction: Neutron vs. X-ray imaging}

Neutron radiography has generally been carried out using strong neutron sources which enable transmission images, mostly based on film exposure using converters. Although many of the aged reactors (and the attached neutron radiography stations) went out of operation in the meanwhile, there are some new installations where state-of-the-art detection systems enable performance similar to what the advanced X-ray systems can deliver. The progress in digital imaging systems has been further used to improve neutron imaging methods onto a level, which can compete with X-ray ones. In particular, digital neutron imaging provides high performance for studies with variable conditions with respect to spatial and time resolutions.

As shown in the example of a sealed Buddha scarifying sculpture (figure 1) both X-ray and neutron transmission images enable different and complementary insights into the object. The high contrast of the X-rays for the metallic (brass) cover is complemented by the opportunity to visualize the organic content (wood, dried plant, fibres, cord) of the sculpture. Both images together can deliver the whole information of the object in completely non-invasive way. In general, neutron imaging has complementary options in comparison to the X-ray methods 

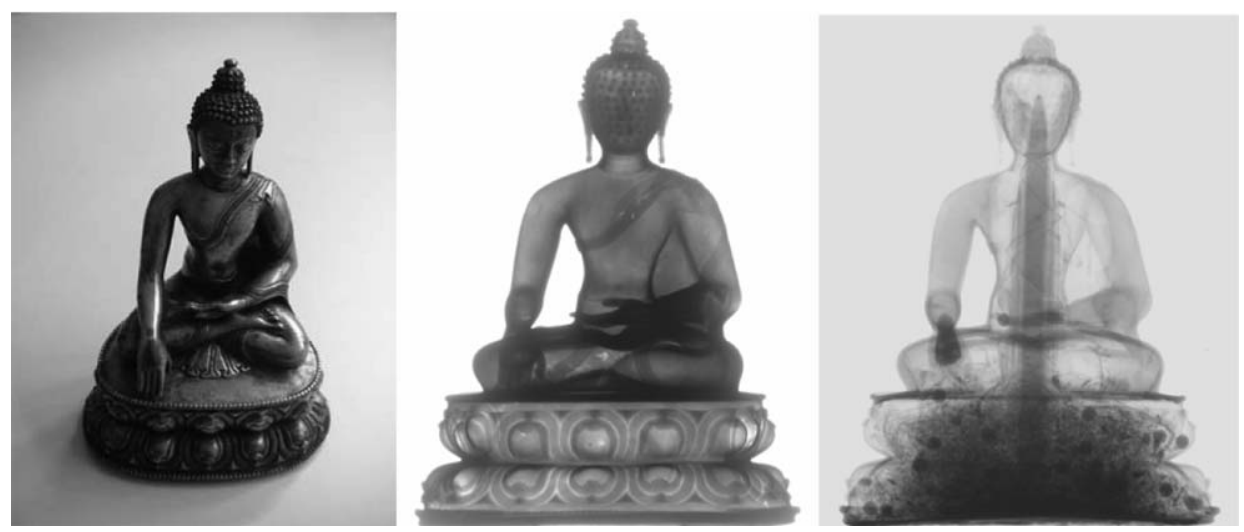

Figure 1. A sealed Buddha figure (left) (about $20 \mathrm{~cm}$ in height, photo) Sakyamuni, Bhumisparsa Mudra, West-Tibet, 14th-15th century - was investigated with $\mathrm{X}$-rays $(150 \mathrm{kV}$ tube voltage, middle) and thermal neutrons (right). Whereas the X-ray image only shows the outer metallic brass cover, neutrons can 'see' also the hidden organic cultic enclosures (wood, dried plants, cord) (courtesy Prof. M O Speidel, CH).

due to the different interaction schemes of either neutrons (thermal or cold) or X-rays.

Whereas neutrons interact with the atomic nuclei, the X-ray attenuation is directly related to the number of electrons in the atomic shell. Accordingly, X-rays have low interaction probability for light elements, but deliver high contrast for heavy materials. Neutron interactions often act reversely to this behaviour.

Competitive neutron image quality can be obtained if a well-developed facility is used. Such installations are available at only few places worldwide [1]. All modern imaging techniques (tomography, real-time imaging, phase contrast enhancement, etc.) are available today for neutrons too, similar to the X-ray techniques in principle, if right installations have been done.

\section{State of the art in neutron imaging}

In order to obtain neutron imaging data with a quality comparable to X-ray techniques, some requirements have to be fulfilled. Despite the high competition for neutron scattering (spectrometers, diffractometers) installations around most neutron sources, it is essential that a dedicated beam line is exclusively used and particularly designed for the neutron imaging applications. The higher the source strength the better is the performance in acquisition time and image quality.

A high beam collimation $(L / D>500)$ is needed to enable best possible image resolution. For some special applications (e.g. phase contrast edge enhancement), the collimation must be even higher. A well-defined thermal or cold spectrum is required to enable quantification and corrections for multiple scattered neutron contributions in the images [2]. Low background from accompanying gamma radiation avoids misleading disturbances in the images. 
Due to the high penetration ability of neutrons for several metals, even large structures can be inspected successfully (turbine blades, casting parts, combustion engines, ceramic insulators etc.). For this purpose, the field-of-view should be as large as possible (beam diameter $>20 \mathrm{~cm}$ ). To enable the investigation, where extended experimental infrastructure inside the well-shielded area is often needed, sufficient space has to be made available around the beam line. Remotely controlled sample manipulators help to perform the studies efficiently and in a reproducible way.

Digital neutron imaging systems are needed for advanced studies. Modern imaging procedures (tomography, real-time studies, phase extraction, quantification) are only possible when used with various digital systems [3].

Although successful neutron imaging needs the technical basis described before, the user program, based on applied science and industrial partnership must be the driving force for the efficient use of the facilities and for further methodical improvements. The systems at PSI (NEUTRA and ICON (see figure 2)) can be used as reference facility [4] as described below.

\section{Facilities at PSI: NEUTRA and ICON}

The native neutron source for research purposes in Switzerland is the continuous spallation source SINQ, in operation since 1997 with success. It compares to a reactor with about $10 \mathrm{MW}$, but has advanced neutron design features like a $\mathrm{D}_{2}$ cold source and supermirror guides for cold neutrons. Modern experimental devices are installed around the target block of the source.

Two beam lines are in use for neutron imaging purposes: NEUTRA at a thermal beam port (since 1997) and ICON at a cold beam port (since 2005).

About 50 to 80 different projects are performed at these facilities per year for customers from the scientific and industrial community. The access to the faculties is organized via a proposal procedure with two deadlines per year.

The major fields of activities are the following for the moment at the PSI installations: welding and soldering of metals, sealing performance, gluing connections within metals and wood, electrochemistry (fuel cells and batteries), objects from cultural heritage, soil-plant interaction, geo- and earth sciences, nuclear fuel and its cladding, running combustion engines, and many more.

\section{Higher spatial resolution}

There is high demand from many customers to increase the presently available 0.1 $\mathrm{mm}$ spatial resolution by about one magnitude at least. There is competition (and complement) from X-ray methods at synchrotron radiation facilities, which can deliver about $0.001 \mathrm{~mm}$ in resolution now. However, the contrast and transmission must be balanced in all cases in respect to the dynamic range of the detection system. Furthermore, it is to consider that the field-of-view relates to the resolution in the order of about 1000 for all systems, given by the number of individual pixels of the detector. 


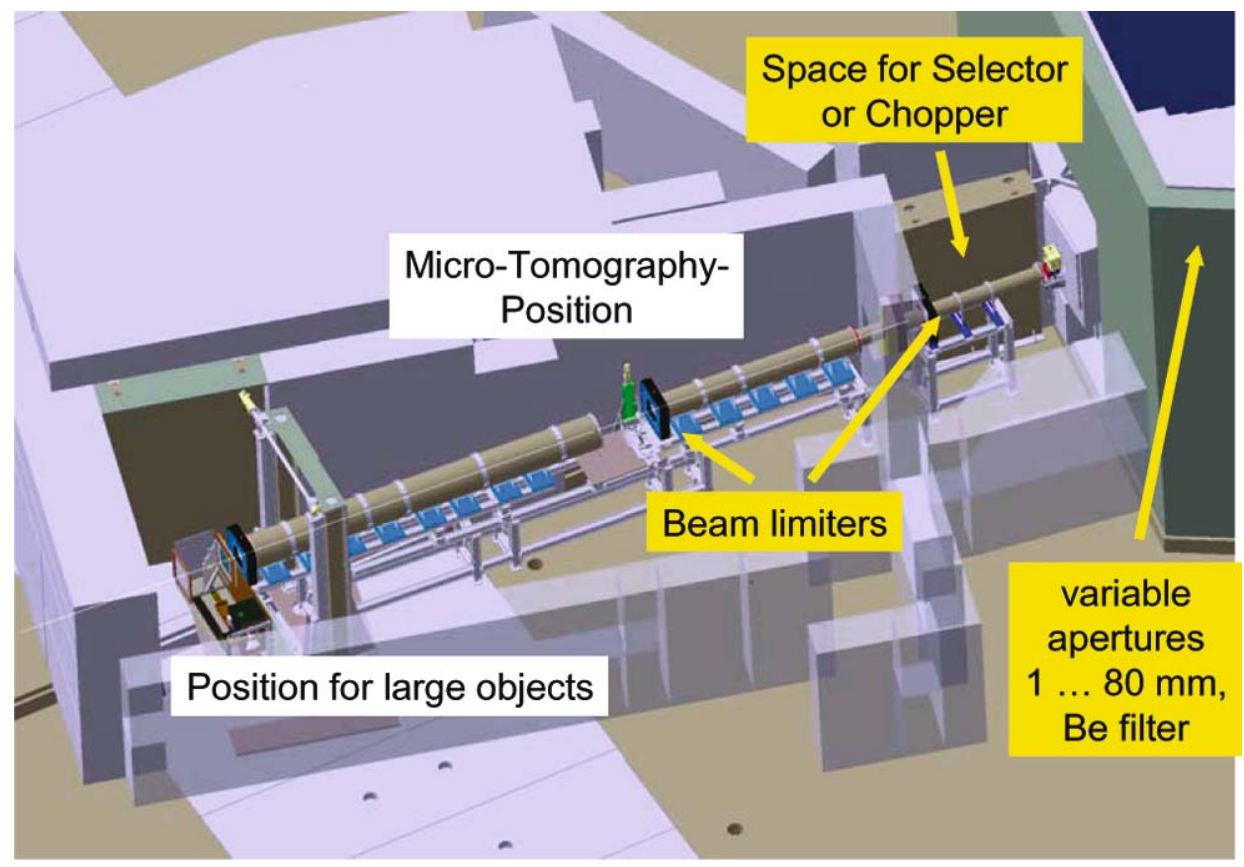

Figure 2. The neutron imaging facility ICON is situated at a cold beam port of the spallation neutron source SINQ (PSI, Switzerland). Due to its flexible construction, neutron imaging investigations can be performed with either high resolution (microtomography set-up) or with a large field-of-view at the end position.

However, the final limitation in resolution is given for neutrons by the range of the secondary particles from neutron interaction in the detection system. Neutrons can only be detected via secondary reaction products from either neutron capture or collision process. It will be very hard to overcome a lower limit of $0.01 \mathrm{~mm}$ in this way. Furthermore, the low number of available neutrons (compared to the very high photon density in advanced synchrotron sources) will limit further improvements in spatial resolution too.

For an improved digital imaging detector at PSI, the following guidelines were applied:

- scintillator screen with lowest possible thickness,

- highest detection probability (ratio capture/light output); an own scintillator screen development was initialized for this purpose,

- cold neutrons for highest possible detector response and sample contrast,

- optical system with superior light transmission, no distortion and limitation in optical resolution (special development together with an optical company),

- CCD camera with $2048 \star 2048$ pixels, $13.5 \mu \mathrm{m}$ each,

- highest possible beam collimation $(L / D>1000)$,

- maximal possible beam intensity. 


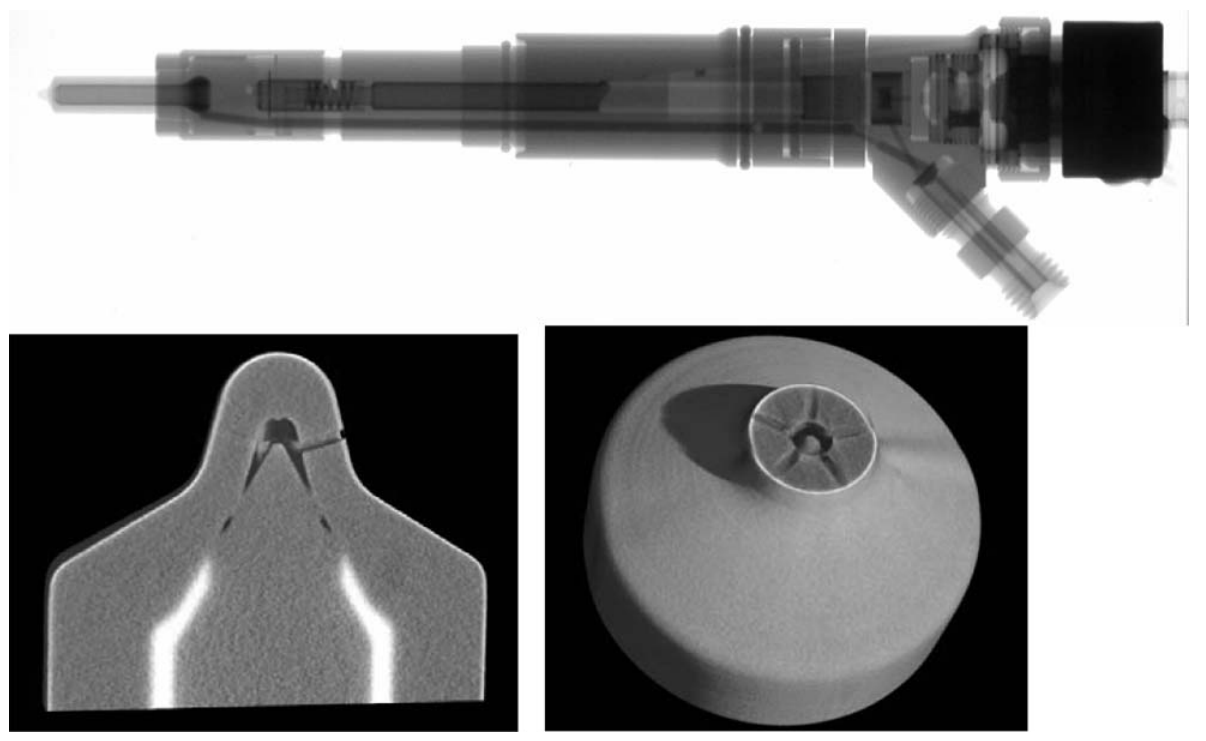

Figure 3. A diesel injection nozzle was investigated with the microtomography option at ICON. The transmission image (top) already shows that the metallic components of the device are more transparent than the diesel oil inside. The two virtual slices (below) through the injection part - very left in the transmission image above - enable to inspect the holes of only $0.15 \mathrm{~mm}$ diameter very precisely.

The resulting system is described in [5] and has already been used in different projects successfully. Results from such experiments are shown in figure 3 .

\section{Energy-selective neutron imaging}

Almost all neutron imaging studies have been performed until now with 'white' thermal or cold beams, where the full spectrum is initially applied and the transmitted neutrons are integrated by the detector according to its spectral response.

It is well-known that polycrystalline metals show large steps in the energydependent cross-section due to the collective scattering at grid planes. These Bragg edges can be exploited by an energy-sensitive neutron imaging procedure to either increase or decrease the transmission contrast for different materials. On the other hand, the energy selection can be used to characterize materials with unknown or changing crystalline behaviour.

Compared to neutron diffractometer studies, which deliver information on the atomic or lattice level, energy-selective neutron imaging can be used for a macroscopic characterization of the samples as a $1: 1$ projection with reasonable spatial resolution ( $0.1 \mathrm{~mm}$ or better).

Another advantage of energy selective imaging with narrow energy bands is its improved option for quantification because spectral changes (beam hardening) will play a less important role. 


\section{Eberhard H Lehmann}

Bragg edges can be found in the cross-section data preferential in the range for cold neutrons $(2-7 \AA)$. Therefore, all practical studies with this approach have to be done with installations at beam line with cold spectrum.

In order to enable narrow energy bands in the cold spectrum, three options for energy selection are available for the moment:

1. Turbine-type energy selector: common at scattering devices (e.g. SANS), where the rotation speed of the turbine $\omega$ and its tilting angle $\theta$ define the transmitted wavelength $\lambda$ according to the equation

$$
\lambda=\frac{h \cdot \theta}{R \cdot m \cdot \varpi},
$$

where $h$ is the Plancks constant, $m$ is the neutron mass and $R$ is the radius, where the neutrons are passing the turbine.

2. Time-of-flight techniques, where pulsed sources are preferred: The time structure of the beam is transferred into an energy structure with the help of a time-dependent detection system. Triggered CCDs have been used successfully for imaging purposes [6].

3. Double-crystal monochromator set-up: a part of the spectrum is sorted out from the initial polychromatic beam at the lattice planes of a first single crystal monochromatizer (graphite, silicon). The second crystal enables to keep the forward direction of the initial beam, when both crystals are turned simultaneously.

These options have similarities: they reduce the initial neutron intensity by magnitudes. Nevertheless, all three options were used to study metallic structures (welds, rolled pieces, composites). One result is shown in figure 4 for a steel weld, where option 3 was applied at the CONRAD facility (BER-2, HMI, Germany). This promising image data are just the first step towards a new imaging technique with great potential in the future.

\section{Imaging at pulsed spallation sources?}

For several reasons (proliferation, waste disposal, missing pulsed options with reactors), all the leading new neutron source projects are based on spallation, in particular pulsed spallation sources. These accelerator-driven devices enable pulses in the $\mu$ s range and repetition frequencies of 20 to $50 \mathrm{~Hz}$. These options are advantageous for all energy-dependent scattering experiments, where the time-of-flight (TOF) principle is used.

For imaging, similar approaches are feasible, although experiences are limited for the moment. The author believes that the energy selection option has much more potential than simple tests can show for the moment.

The combination of transmission and diffraction is very promising for material research and other studies. Projects with such combinations are started already.

However, a dedicated and well-designed beam line is needed for the best possible approach. Beside the field for energy-selective investigations, all 'standard' applications can be made possible as well using the integration mode. 

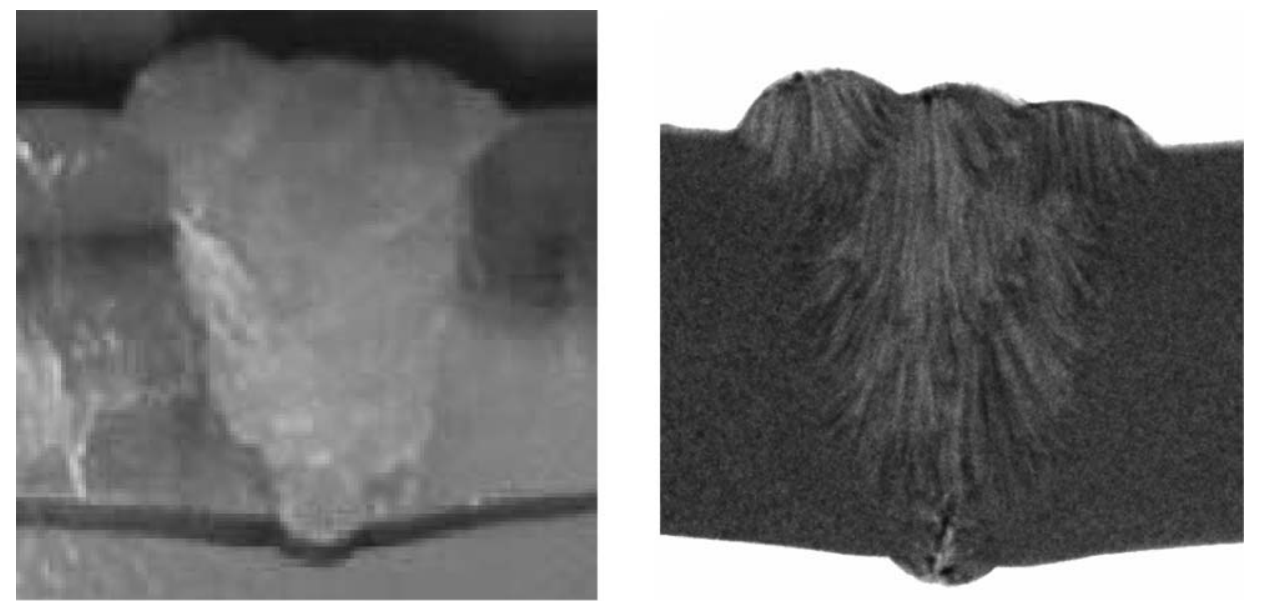

Figure 4. Study of a steel weld (left - photo, right - transimission neutron image) with the energy-selective neutron imaging option using a double monochromator set-up at HMI (facility CONRAD [7]). The field-of-view is 27 $\mathrm{mm}$, the energy is $3.9 \AA$ and the energy width is $0.1 \AA$. Compared to the bulk material, microcrystalline structures become visible at certain energy bands.

Furthermore, a stroboscopic option will become important to study timedependent and repetitive processes of industrial importance (engines, pumps, turbines etc.).

\section{Further aspects and options}

Many new features in the non-destructive study with radiation (X-rays, neutrons) have been developed in the past years. All kinds of digital imaging, including tomography and image referencing have become routine in advanced laboratories. Nevertheless, there is still potential for further improvements.

It is of high importance that these modern methods be adapted to the practical needs of the scientific and industrial users (micro-tomo, phase-contrast, energyselective imaging, X-ray referencing).

Therefore, an improved and dedicated communication with industrial partners is needed in order to satisfy their specific demands. On the other hand, the best possible infrastructure should be established for test runs, where the costs for the installations have to be shared among the partners.

A further combination of imaging and scattering methods accompanied by a deeper understanding of the principles from both sides should be the most promising trend for the future.

One promising technique in neutron imaging (as in X-ray applications) is the use of the phase information for image enhancement (as shown in figure 5) or as a separate signal [8]. 


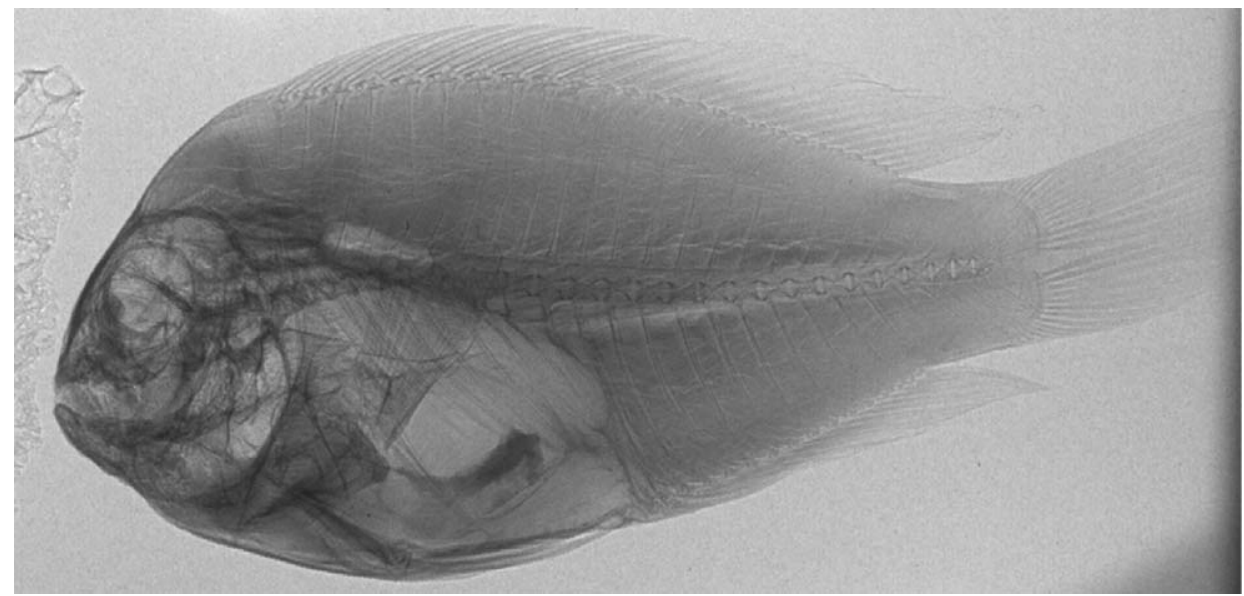

Figure 5. Phase contrast edge enhancement in the example of a dried fish (about $5 \mathrm{~cm}$ in length). The improvement in the contrast has been obtained by a $1 \mathrm{~mm}$ pinhole aperture, a sample-detector distance of $0.7 \mathrm{~m}$ and a source-sample distance of $10 \mathrm{~m}$. Using an imaging plate detector, the exposure time was about $1 \mathrm{~h}$.

$$
n=1-\delta+i \beta=1+\lambda^{2} \frac{N}{2 \pi} \sqrt{b_{\mathrm{c}}^{2}-\left(\frac{\sigma_{\mathrm{t}}}{2 \lambda}\right)^{2}}+i \frac{\sigma_{\mathrm{t}} N \lambda}{4 \pi},
$$

where $N$ is the number of sample atoms, $b_{\mathrm{c}}$ is the coherent scattering length [9], $\sigma_{\mathrm{t}}$ is the total cross-section and $\lambda$ is the neutron wavelength.

This formula describes the neutron interaction with matter, where the real part of the refractive index belongs to the phase shift and the imaginary one to neutron absorption. Even if $\delta$ is very small for most materials (in the order of $10^{-6}$ ), there are dedicated methods for its determination as image for the sample under investigation.

\section{Acknowledgements}

First of all, the author deeply acknowledges the impressive results of the team at the PSI imaging installations, in particular by G Frei, P Vontobel, G Kühne and S Hartmann. Major impacts to the energy-selective experiments were given by W Kockelmann (ISIS, RAL, UK) and J Santisteban (Bariloche, Argentina). The studies at HMI together with N Kardjilov, A Hilger and M Strobl enabled alternative insights into the neutron interaction with matter at well-defined energy ranges.

\section{References}

[1] E Lehmann, Neutron Imaging methods and their applications, in: Neutron applications in earth, energy and environmental sciences (Springer Sci. Publ., 2008) in preparation 
[2] R Hassanein, Correction methods for the quantitative evaluation of thermal neutron tomography, Dissertation No. 16809, ETH Zurich, Switzerland, 2006

[3] E H Lehmann et al, Nucl. Instrum. Methods in Phys. Res. A531, 228 (2004)

[4] E Lehmann, P Vontobel, L Wiezel, Nondestr. Test. Eval. pp. 100-113 (2000)

[5] E H Lehmann et al, Nucl. Instrum. Methods in Phys. Res. A576(2-3), 389 (2007)

[6] W Kockelmann et al, Nucl. Instrum. Methods in Phys. Res. A578, 421 (2007)

[7] W Treimer, M Strobl, N Kardjilov, A Hilger and I Manke, Appl. Phys. Lett. 89, 203504 (2006)

[8] F Pfeiffer, C Gruenzweig, O Bunk, G Frei, E Lehmann and C David, Phys. Rev. Lett. 96, $215505(2006)$

[9] http://www.ncnr.nist.gov/resources/n-lengths/ 